\title{
On en revient une nouvelle fois à Osler: «Osler's Ten»
}

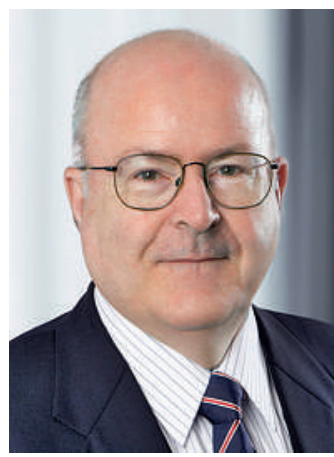

Werner Bauer
Certains lecteurs vont s'irriter de ce titre: l'auteur de ces colonnes n'a-t-il donc vraiment plus d'autres sources d'inspiration? D'autres parmi vous concluront avec amusement qu'Osler est décidément le cheval de bataille du rédacteur devenu lunatique et espéreront que ce dernier finira par en enfourcher un autre. Quelques-uns s'interrogeront pourtant peutêtre sur la particularité des «Osler's Ten».

L'article principal du bulletin 1/12 de l'Académie Suisse des Sciences Médicales ASSM (M. Louis-Courvoisier) porte le titre «Les Sciences humaines en médecine: un plaidoyer pour la plasticité intellectuelle». $\mathrm{Au}$ motif que les Medical humanities peuvent, outre les connaissances techniques, largement contribuer au développement de la personnalité d'un médecin (l'être-médecin), on encourage l'introduction croissante et délibérée des sciences humaines et sociales, de la littérature et des arts visuels dans la formation médicale. Même si les catalogues d'objectifs d'apprentissage sont déjà pleins à ras bord, l'idée que les sciences humaines peuvent induire des formes de raisonnement utiles et importantes dans le cadre du suivi des patients et d'autres activités professionnelles dans la recherche et le secteur de la santé, semble gagner du terrain aux Etats-Unis et désormais également en Europe. Dans son éditorial, le Président de l'ASSM, l'exprime ainsi: «Les questionnements liés à la médecine et la biologie modernes ne peuvent être résolus, dans le respect de l'homme et de la société, sans tenir compte des aspects des sciences sociales et humaines.»

L'équipe de spécialistes chargée de l'accréditation de la Faculté de Médecine de Berne a émis un jugement très favorable dans sa présentation finale, mais a également suggéré de creuser la piste d'une multiplication des opportunités de contact des étudiants avec les sciences humaines.

Ce qui nous ramène au point de vue étonnamment actuel de Sir William Osler:

"Well filled though the day be with appointed tasks, to make the best possible use of your one or of your ten talents, rest not satisfied with this professional training, but try to get the education, if not of a scholar, at least of a gentleman. Before going to sleep read for half an hour, and in the morning have a book open on your dressing table. You will be surprised to find how much can be accomplished in the course of a year. I have put down a list of ten books which you may make close friends. There are many others; studied carefully in your student days these will help in the inner education of which I speak (Aequanimitas).»

Voici les dix œuvres très variées sélectionnées par Osler en tant que «bedside library for medical students»:

- Plutarque: Vitae parallelae

- Thomas Browne: Religio Medici

- William Shakespeare: Othello, Songe d'une nuit d'été, Hamlet, Les Sonnets

- Michel de Montaigne: Essais

- Marc Aurèle: Méditations

- Epictète: CEuvres

- Miguel de Cervantes: Don Quichote

- Ralph Waldo Emerson: Essays and Lectures

- Oliver Wendell Holmes: The autocrat of the breakfast-table

- Ancien et Nouveau Testament

Il ne s'agissait pas pour Osler d'une liste de «best-sellers» pour étudiants en médecine, car il évoque également, dans ses écrits et lors de ses conférences, toute une série d'autres auteurs l'ayant marqué (de Charles Dickens à Pascal, en passant par Voltaire, Ibsen et Avicenne).

L'essentiel pour lui était la conviction que la lecture d'œuvres majeures de la littérature mondiale était susceptible d'élargir considérablement le champ de réflexion des jeunes médecins. Il l'a résumé ainsi: «The practitioner needs culture as well as learning."

D'Osler à nos jours: je souhaite qu'il y ait autant de jeunes et de futurs médecins que possible intéressés par la littérature, la langue en tant que base de raisonnement et qu'instrument de guérison, les arts visuels, la philosophie et les autres sciences humaines. Je souhaite en outre que la formation initiale et complémentaire compte des responsables qui se sont constitu une sorte de «liste des dix» (mais il peut y en avoir plus) et qui trouvent en outre parfois le temps de discuter avec les étudiants et les internes d'idées qui leur sont venues en lisant. Si, parmi les lectrices ou les lecteurs, l'un(e) ou l'autre avait des suggestions pour une liste des «Osler's Ten 2012», cela m'intéresserait grandement.

Werner Bauer* 